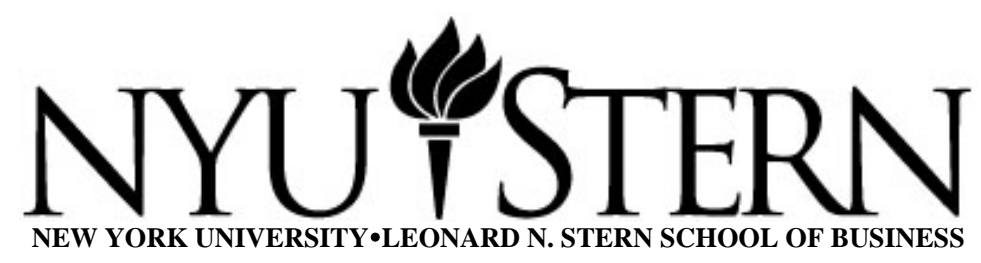

Department of Finance

Working Paper Series

FIN-03-009

\title{
An Integrated Pricing Model for Defaultable Loans and Bonds
}

\author{
Mario Onorato and Edward I. Altman
}

February 2003

The 2003 NYU Stern Department of Finance Working Paper Series is generously sponsored by

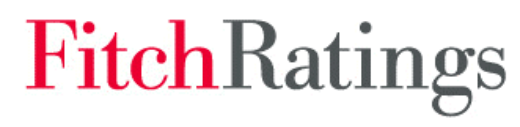




\section{An Integrated Pricing Model for Defaultable Loans and Bonds}

\author{
Mario Onorato ${ }^{1}$ \\ Cass Business School, City University \\ 106, Bunhill Row \\ London, EC2Y 8TZ, U.K. \\ Phone: + 442070408698 \\ e-mail: M.F.Onorato@city.ac.uk
}

\author{
Edward I. Altman ${ }^{2}$ \\ NYU Stern School of Business \\ 44 west Street \\ New York, NY 10012, \\ USA \\ Ph.: 212 998-0709 \\ E-mail:altman@stern.nyu.edu
}

\footnotetext{
${ }^{1}$ M. Onorato is Research Fellow at Cass Business School, London City University and Visiting at Erasmus University Rotterdam

${ }^{2}$ E. Altman is the Max L. Heine Professor of Finance, NYU Stern School of Business and Vice Director of the NYU Salomon Center
} 


\title{
An Integrated Pricing Model for Defaultable Loans and Bonds ${ }^{1}$
}

\author{
JEL classification: C15, C69, G12, H63
}

Keywords: statistical simulation methods, financial risk management, credit risk measurement model, asset pricing, debt \& debt management.

\begin{abstract}
In recent years, credit risk has played a key role in risk management issues. Practitioners, academics and regulators have been fully involved in the process of developing, studying and analysing credit risk models in order to find the elements which characterize a sound risk management system. In this paper we present an integrated model, based on a reduced pricing approach, for market and credit risk. Its main features are those of being mark to market and that the spread term structure by rating class is contingent on the seniority of debt within an arbitrage-free framework. We introduce issues such as, the integration of market and credit risk, the use of stochastic recovery rates and recovery by seniority. Moreover, we will characterise default risk by estimating migration risk through a "mortality rate", actuarial based, approach. The resultant probabilities will be the base for determining multi-period riskneutral transition probability that allow pricing of risky debt in the trading and banking book.
\end{abstract}

\footnotetext{
${ }^{1}$ We thank Giovanni Barone Adesi, Winfried Hallerbach, Anthony Saunders, Jaap Spronk, Rangaraian Sundaram, Constantine Thanassoulas, three anonymous referees, and seminar participants at Italian Banking Association for helpful discussion of this study. Earlier versions of this paper were presented at the Annual Meetings of both Financial Management Association (Paris, 2001) and European Financial Management Association (London, 2002) and at the Euro Working Group on Financial Modelling (Capri, 2002).
} 


\section{Introduction}

The 1988 Basle Capital Accord, which created a minimum risk-based capital adequacy requirement for banks, marked a major step forward in introducing risk differentiation into the regulatory framework but did not represent the "optimum" solution. The 1996 amendment to the Capital Accord aimed to correct some of the issues concerning the original accord, but it did not change the section regarding credit risk. As a consequence, regulatory capital for credit assets was still not an appropriate basis for the capital allocation process. Financial institutions started developing their own internal credit risk systems using economic capital. In April 1999, regulators proposed a document, Credit Risk modelling: current practice and applications ${ }^{[6]}$, which aimed at assessing the potential applications and limits of credit risk models ${ }^{2}$ for supervisory and regulatory purposes, in sight of the foreseeable amendment to the Capital Accord. The Basle Committee proposed the amendment in June 1999: A new capital adequacy framework $^{[8]}$ (which is expected to be finalised in 2003). This event marked a breakthrough as regards the credit risk concept for capital adequacy. The committee's proposal dramatically modified the standard approach for capital requirements imposed by the 1988 Accord. A more realistic approach was introduced based on internal rating systems. Moreover, it presented the reclassification of securities taking into account credit risk in all its aspects: default, migration, recovery rates, credit spreads, aggregation and concentration risk. Over the last decade risk managers, regulators, academics and software vendors are devoted to define a sound credit (and market) risk measurement and management system. The main objective of this study is to define a general framework to price risky debt. The pricing of any risky security must reflect the return on a risk-free asset plus a risk margin. The risk margin must compensate the investor for the risk assumed which is represented by: both the transition and recovery (by seniority of debt) risk, liquidity risk, credit exposure risk, default correlation risk, collateral risk and concentration risk. Moreover, in an integrated framework, another source of risk has to be considered, namely: market and credit correlation risk $^{3}$. There are several approaches, which may be used to jointly model interest rates and credit spreads. In this paper reduced ${ }^{4}$ form models and in particular the ones directly modelling credit spread components (transition and recovery risk) are considered. Our approach is a generalization of the Das \& Tufano ${ }^{[22]}$ (DT) (1996) model, which is an extension of the Jarrow-Lando-Turnbull (JLT) (1997) ${ }^{[35]}$ model, and uses credit ratings to characterize the transition risk. Unlike the JLT model, the DT model makes the recovery rate in the event of default stochastic, and provides a two-factor decomposition of credit spreads. In this paper we generalise this approach by considering different set-ups for different seniority classes of debt. Therefore, its main features are those of being mark to market (MTM) $)^{5}$ and that the spread term structure by rating class (STSRC) is adjusted for a spread term structure which is contingent on the seniority of debt (SSD) within an arbitrage free framework. Summarising, in our integrated pricing model we take into account the risk coming from both interest rates variations and credit event verifications ${ }^{6}$. The remainder of this paper is organised as follows. Section 2 defines default and transition risk and

\footnotetext{
2 The document issued by the Basle Committe ${ }^{[6]}$ analyses in particular four widespread credit risk models: Creditmetrics $^{\mathrm{TM}}$ (JP Morgan 1997) ${ }^{[15]}$; KMW Portfolio Manager ${ }^{\mathrm{TM}}$ (Kealhofer 1998) ${ }^{[17],[38],[39]}$, CreditRisk ${ }^{\mathrm{TM}}$

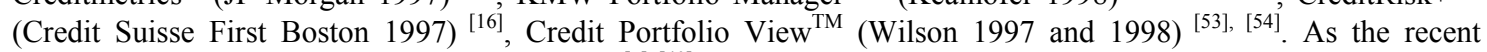
literature shows Koyluoglu and Hickman (1998) ${ }^{[5],[40]}$, these models fit within a single generalised framework..

${ }^{3}$ Consequently, all the correlations among the market factors (which drive the price of securities) and credit risk factors-also called background factors- which affect the creditworthiness of obligors in the portfolio, need to be identified and modelled. For an illustrative description of the background factors within the credit risk model framework refer to Koyluoglu and Hickman $(1998)^{[40]}$ and Wilson(1997 and 1998) ${ }^{[53],[54]}$.

${ }^{4}$ Reduced form models are so called to contrast them to structural models (Merton $1974^{[44]}$ ). The difference between structural and reduced form model is outlined in section 2 and 4. For a complete review of the inherent literature refer to the work of Acharya, Das and Sundaram (2002) $)^{[1]}$.

5 "In contrast to the default mode paradigm, within the mark to market (or, to be more accurate, mark to model) paradigm a credit loss can arise in response to deterioration in asset's credit quality shorts of default". Foe a detailed discussion on the topic please refer to the work of Basle Committee, pag. $21^{[6]}$.

${ }^{6}$ When a credit event occurs the credit quality of the issuer changes. Examples of such events are given in section 2.
} 
it is aimed at illustrating both the most common methods to estimate them and the inherent empirical evidence. Section 3 analyses the same issues for the recovery rate by seniority of debt risk. Section 4 is the core of the paper. In this section the theoretical integrated pricing model is illustrated. The bulk of the suggested approach relies on the modelling of both the stochastic spread term structure by rating class and the spread contingent to the seniority of debt within a unified arbitrage-free framework. Section 5 illustrates possible applications of the integrated pricing model. Section 6 concludes.

\section{Default and Transition Risk}

Credit pricing and risk models attempt to measure credit losses. Losses are the consequence of the firm's financial position and asset quality deterioration, which then leads to the degradation of its creditworthiness (credit migration). The determination of the creditworthiness of the issuer is difficult as it is driven by many factors such as general economic conditions, industry trends and specific issuer factors like the issuer's financial wealth, leverage, market value, equity value, asset value, capital structure and less tangible things such as reputation and management skills.

The probability of a customer migrating from its current risk-rating category to any other category, within a pre-defined time horizon, is frequently expressed in terms of a rating transition matrix ${ }^{7}$. Rating migration probabilities are therefore collected in the transition (migration) matrices and describe the probability of migrating from any given credit rating to another one. Moreover, estimates of transition probabilities often suffer from small samples, either in the number of rated firms or in the number of events; in particular this happens when considering transition towards the most "distant" rating classes. This often results in biased estimates of these types of transition probabilities that have led the Basle Committee on Banking Supervision to impose a lower minimum probability of 0.0003 for rare events. In our analysis we will cluster obligors into obligors rating classes. In this matrix the worst internal grade corresponds to the worst state, that is the default state (last column of the matrix) ${ }^{8}$. Let us assume to deal with $K$ rating classes (the $K$ th being the default state), then, the transition matrix is the collection of one-step transition probabilities of migrating from any class- $i$ to any class $-j$ at the given time- $m$, including the probabilities of remaining in the same class (corresponding to the off-diagonal values). The statistical, or actual, probabilities matrix can be represented as:

$$
\bar{Q}(m)=\left\{\bar{q}_{i j}(m)\right\}=\left[\begin{array}{cccc}
\bar{q}_{11}(m) & . . & . . & \bar{q}_{1 K}(m) \\
\bar{q}_{21}(m) & \bar{q}_{22}(m) & . . & \bar{q}_{2 K}(m) \\
. . & . . & . . & . . \\
\bar{q}_{K 1}(m) & \bar{q}_{K 2}(m) & . . & \bar{q}_{K K}(m)
\end{array}\right] \equiv\left[\begin{array}{cccc}
\bar{q}_{11}(m) & . . & . . & \bar{q}_{1 K}(m) \\
\bar{q}_{21}(m) & \bar{q}_{22}(m) & . . & \bar{q}_{2 K}(m) \\
. . & . . & . . & . . \\
0 & 0 & 0 & 1
\end{array}\right]
$$

Once the default state is reached, no other rating classes are possible to exist at the next time step, therefore all the transition probabilities are defined to be null, with the exception of the probability of staying in the $K$-state i.e. default. As a consequence of the definition of transition probability, another relevant property of the migration matrix is that the sum over the elements of the same row must equal one: $\sum_{j=1}^{K} \bar{q}_{i j}(m) \equiv 1 ; \forall i$. In the integrated model, the statistical transition probabilities are derived from empirical data by using the mortality approach briefly described in paragraph 2.1. The process determining customer defaults or rating migrations can be modelled through two approaches: actuarial based methods and equity based methods.

\footnotetext{
${ }^{7}$ See Altman, Caouette and Narayanan $(1998)^{[2]}$ for a discussion on this topic.

8 In a two state default process, within the considered time horizon, there are only two possible events: "no default" and "default".
} 


\subsection{Actuarial based method}

The basic actuarial approach uses historical data on the default rates of borrowers to predict the expected default rates for similar customers. The actuarial (also called empirical) model is based on the estimation of statistical (or actual) transition probabilities ${ }^{9}$. This method uses historical data to evaluate the migration probabilities. In this model the inputs are represented by empirical data and the output will be the statistical estimation concerning these data. One of the most important criticisms to the empirical approach is the apparently static nature of the resulting average historical probabilities. In reality, actual transition and default probabilities are very dynamic and can vary quite substantially over one year, depending on general economic conditions and business cycles ${ }^{10}$.

In our integrated pricing model we will estimate the actual transition probability by using the Altman (1998) mortality rate approach ${ }^{[2]}$. This method determines the (expected) default rate, using an empirical method. An important element that needs to be observed is the aging effect ${ }^{11}$, that is, the time between instruments' issue up to valuation time. This approach implies lower default probabilities in the first year than in the next years. The question is not whether a bond or another credit derivative is going to default or not but when it is going to default and what will be the likely recovery, given its original rating and its original seniority. Credit instruments are classified for issue time and rating classes. After this classification it is possible to calculate the default probability. In order to do this, it is necessary to calculate the marginal mortality rate and the cumulative mortality rate.

The marginal mortality rate (MMR) is the probability that a credit instruments defaults over the first year, over the second and so on. The MMR can be expressed both in term of number and value. In the last case MMR is equal to the ratio between the total (nominal) value of the corporate bonds included in a specific rating class defaulting over the planning horizon and the total (nominal) value of corporate bonds included in the same rating class, at the beginning of the time horizon.

$$
M M R_{i}=\frac{\text { Total value of defaulted bonds in the } i_{\text {th }} \text { year }}{\text { Total value of bonds issued at the beginning of the } i_{\text {th }} \text { year }}
$$

where $i=1 \ldots \mathrm{NN}=$ number of years ${ }^{12}$

Consequently the survival rate $\left(\mathrm{SR}_{\mathrm{i}}\right)$ is equal to $\mathrm{SR}_{\mathrm{i}}=1-\mathrm{MMR}_{i}$.

One can measure the cumulative mortality rate $\left(\mathrm{CMR}_{\mathrm{T}}\right)$ during a specific time period, subtracting the product of the surviving populations over the previous time, that is $\mathrm{CMR}_{\mathrm{T}}=1$ $\prod_{i=1}^{T} S R_{i}$. Altman $^{[2]}$ derives the migration probabilities for each rating class from $\mathrm{CMR}_{\mathrm{T}}$, which represents the default probability.

\footnotetext{
${ }^{9}$ Here we refer to actual (statistical or empirical) transition probability in contrast to risk-neutral probability

${ }^{10} \mathrm{~A}$ real dilemma concerns the private companies that are neither rated by the agencies nor publicly traded. In fact a substantial proportion of these portfolios do not have very clear benchmark for estimating default and transition probabilities

${ }^{11}$ Altman's method (1998) is different from other methods determining the "aging effect". In fact Moody's and S\&P use static pools (including all credit instruments), while Altman makes a distinction among instruments according to the issue date. The (actual) transition probability matrix in the integrated pricing model can be easily inferred from the migration matrix, which is estimated using the Altman mortality rate approach. For a more detailed illustration of the mortality (default) rate by rating and by age approach please refer to Altman $(1998)^{[2]}$

${ }^{12}$ If, for example, the par vale outstanding of high-yeld debt in 1997 was 335.400 (\$ millions) and the par value defaults was 4.200 (\$ millions), the MMR (or alternatively the default rates) was $1.252 \%$
} 


\subsection{The equity based method}

The equity-based approach, often associated with the Merton model ${ }^{[44]}$, is mainly used for estimating the Expected Default Frequencies (EDF) ${ }^{13}$ of large and middle-market business customers, and is often used to crosscheck estimates generated by actuarial-based methods. This technique uses publicly available information on a firm's liabilities, the historical and current market value of its equity and the historical volatility of its equity to estimate the level, rate of change and volatility (at an annual rate) of the economic value of the firm's assets. There are at least three practical limitations to implement the option (Merton model) approach:

1. It is necessary to know the market value of firm's asset. This is rarely possible as the typical firm has numerous complex outstanding debt contracts traded on an infrequent basis.

2. It is necessary to estimate the return volatility of the firm's asset. Since the market prices cannot be observed for the firm's assets, the rate of return cannot be measured and volatilities cannot be computed.

3. It is necessary to simultaneously price all the different types of liabilities senior to the corporate debt under consideration. Most corporations have complex liabilities structures $^{14}$.

Summarizing, the key ingredient of credit asset pricing and risk modelling is the default (or, in a multi-state framework, transition) risk, which is the uncertainty underlying a firm's ability to service its debts and obligations. Prior to default, there is no way to discriminate unambiguously between firms that will default and those that will not. At best we can only make probabilistic assessments about the default possibility. In practice, we use transition probabilities basically for two main reasons:

1. In the trading book, to price credit sensitive instruments adjusting it through the risk neutral transition probability

2. In the banking book, to measure the credit risk of portfolio losses of loans

\section{Recovery by seniority of debt risk}

The default is one of the main types of credit events that determine loss amount occurring once a credit has defaulted. This credit loss, also called loss given default (LGD) is defined as the difference between the bank's credit exposure and the present value of the future net recoveries (cash payments from the borrower less workout expenses). Therefore the recovery rate (RR) is equal to the ratio between 1-LGD and the initial exposure ${ }^{15}$. LGD depends on a limited set of variables characterising the structure of a particular credit facility. These variables may include the type of product (e.g. business loan or credit card loan), its seniority, collateral and country

\footnotetext{
${ }^{13}$ Expected default probabilities can be inferred from the option models under the assumption that default occurs when the value of a firm's assets falls below its liabilities. See Crosbie $(1998)^{[17]}$ for a detailed description of how the EDF are estimated within the KMV model.

${ }^{14}$ For an interesting and detailed analysis of the limitations of the equity approach see Jarrow and Turnbull (2000) ${ }^{[36]}$

${ }^{15}$ The estimation of LGD depends on the availability of historical loss data that may be retrieved by the following possible sources: bank's own historical LGDs records, samples by risk segment; trade association and publicly available regulatory reports; consultants' proprietary data on client LGDs, and published rating agency data on the historical LGDs of corporate bonds.
} 
of origination. In the Credit Risk $+{ }^{\mathrm{TM}}[16]$ model $^{16}$ the LGD is treated as a deterministic variable while in the other structural models is treated as a random variable ${ }^{17}$. Reduced form model assume either a constant (Litterman and Iben ${ }^{[41]}, \mathrm{JLT}^{[35]}$, for example) or a stochastic recovery rate (Duffie and Singleton ${ }^{[28]}$ and $\mathrm{DT}^{[22]}$ for example). These models assume zero correlation among the LGDs of different borrowers, and hence no systematic risk due to LGD volatility ${ }^{18}$. Moreover, the empirical evidence shows that the recovery rates are both state ${ }^{19}$ and structure ${ }^{20}$ dependent. In particular, our analysis based on the last three decades default and recovery data on US corporate bonds shows that the recovery rate changes depend on the seniority of debt. In fact, comparing senior secured and unsecured bonds one can see that the recovery distribution for the latter is more spread out and has a longer lower tail (see table 1$)^{21}$.

Recovery Rates by Seniority and Original Bond Rating, 1971-2001

\begin{tabular}{|c|c|c|c|c|c|}
\hline \multirow[b]{2}{*}{ Seniority } & \multirow[b]{2}{*}{$\begin{array}{c}\text { Number of } \\
\text { Observations }\end{array}$} & \multicolumn{3}{|c|}{ Recovery Rate } & \multirow[b]{2}{*}{$\begin{array}{l}\text { Standard } \\
\text { Deviation }\end{array}$} \\
\hline & & $\begin{array}{c}\text { Average } \\
\text { Price } \\
\end{array}$ & $\begin{array}{c}\text { Weighted } \\
\text { Price } \\
\end{array}$ & $\begin{array}{c}\text { Median } \\
\text { Price } \\
\end{array}$ & \\
\hline \multicolumn{6}{|l|}{ Senior Secured } \\
\hline Investment Grade & 35 & $\$ 62.00$ & $\$ 66.00$ & $\$ 56.88$ & $\$ 19.70$ \\
\hline Non-Investment Grade & 113 & 38.65 & 32.89 & 30.00 & 29.46 \\
\hline \multicolumn{6}{|l|}{ Senior Unsecured } \\
\hline Investment Grade & 159 & $\$ 53.14$ & $\$ 55.88$ & $\$ 50.00$ & $\$ 26.14$ \\
\hline Non-Investment Grade & 275 & 33.16 & 30.17 & 31.00 & 25.28 \\
\hline \multicolumn{6}{|l|}{ Senior Subordinate } \\
\hline Investment Grade & 10 & $\$ 39.54$ & $\$ 42.04$ & $\$ 27.31$ & $\$ 24.23$ \\
\hline Non-Investment Grade & 283 & 33.31 & 29.62 & 28.00 & 24.84 \\
\hline \multicolumn{6}{|l|}{ Subordin ated } \\
\hline Investment Grade & 10 & $\$ 35.64$ & $\$ 23.55$ & $\$ 35.69$ & $\$ 32.05$ \\
\hline Non-Investment Grade & 206 & 31.73 & 28.87 & 28.00 & 22.06 \\
\hline
\end{tabular}

Table 1 . Recovery rates by seniority and original rating

\footnotetext{
${ }^{16}$ For portfolios characterised by distributions of exposure sizes that are highly skewed, the assumption that LGDs are known with certainty may tend to bias downwards the estimated tail of the PDF of credit losses

${ }^{17}$ In these models the LGD probability distribution is assumed to take the form of a beta distribution because this result in a type of distribution whose shape is tipically skewed to the right as shown in the empirical works of Altman and Kishore (1996) ${ }^{[2]}$, Carty and Lieberman (1996) ${ }^{[10],[11]}$, Duffie and Singleton (1996) ${ }^{[28]}$, Castle, Keisman and Yang $(2000)^{[13]}$

${ }^{18}$ Furthermore, they assume independence among LGDs associated with the same borrower. The assumption that LGDs between borrowers are mutually independent may represent a serious shortcoming when the bank has significant industry concentrations of credits. Furthermore, the independence assumption is clearly false with respect to LGDs associated with similar (or equally ranked) facilities to the same borrower. The assumption of default intensities independence may contribute to an understatement of losses to the extent that LGDs associated with borrowers in a particular industry may increase when the industry as a whole is under stress.

${ }^{19}$ Some evidence consistent with the state-dependence of recovery rates is presented in the analysis, based on recovery rates, compiled by Moody's for the period 1974 through 1996 (Carty and Lieberman, 1996 ${ }^{[10], ~[11]}$ ). However, even for senior secured bonds, there was substantial variation in the actual recovery rates. Although these data are also consistent with cross-sectional variation in recovery that is not associated with stochastic variation in time of expected recovery, Moody's recovery data also exhibit a pronounced cyclical component. There is equally strong evidence that of corporate bonds vary with the business cycle (as is seen, for example, in Moody's data) Speculative-grade default rates tend to be higher during recessions, when interest rates and recovery rates are typically below their long-run means.

${ }^{20}$ See Castle, Keisman and Yang (2000) ${ }^{[13]}$

${ }^{21}$ Source: Altman and Pompeii (2002) ${ }^{[13]}$.Also Duffie and Singleton $(1998)^{[28]}$ found similar results.
} 
The analysis also ranks the results in investment grade and non-investment grade. In fact, when evaluating an instrument at the first steps of its life the type of guarantee rate on the underlying security is an extremely relevant characteristic, which - according to historical data - implies that the credit is subject to a global lower risk. This is shown in Table 1, where the rating class, at the time of issuance, non-investment grade in particular, is not influencing the average values as much as the seniority class does (see the senior secured and senior unsecured investment grade case). The most relevant issue is that the dominant factor influencing the evaluation of the security is the composition of both the recovery rate and default probability. Actually, low default rates do not assure that in case of default the recovery rate is low as well; on the other hand high recovery rate is not a credit low-risk index alone, since the security might by highly defaultable, implying the elevated investment risk. In the integrated model we will estimate spread term structure by rating class, to explicitly consider the credit rating (risk) transition and will correct them by means of spread term structure of recovery rate by seniority of debt, both in a arbitrage free framework, in order to get a risk-neutral price of the financial instruments.

\section{The theoretical integrated pricing model}

There are two main approaches to pricing credit risky instruments: the structural ${ }^{22}$ and the reduced form approach. It is argued that structural approaches are of limited value when applied to price interest rate and credit sensitive instruments and, consequently, in measuring and managing market and credit risk in an integrated fashion. Rosen $(2002)^{[47]}$ shows that since the main focus of the structural model is the measure of the counterparty exposure risk, they assume deterministic market risk factors, such as interest rate risk. In contrast to structural models, which assume a specific microeconomic process generating customers' default and rating migrations, reduced-form models attempt to directly describe the arbitrage free evolution of risky debt values without reference to an underlying firm-value process. Acharya, Das and Sundaram (2002) $)^{[1] 23}$ show how this class of model has resulted in successful conjoint implementations of term-structure models with default models. The objective pursued within the suggested integrated pricing model is that of deriving a general framework for pricing risky debt, both plain vanilla (as for example corporate bond) and (credit) derivative. Present values of all cash flows are calculated by using both stochastic interest rate term structure (market risk) and stochastic credit spread term structure (credit risk). This last term can be decomposed in the following risk sources: 1) stochastic recovery rates by seniority, 2) correlation between interest rate term structure and stochastic recovery rates (correlation of market and credit risk) and 3) multi state transition probability at the $m{ }^{\text {th }}$ time step for the $M$-period process. In this set up the proposed integrated pricing model may be considered a multi-period mark to model framework. As for all reduced-form models, also in our integrated model we start modelling the risk free term structure by considering an underlying process for the evolution of risk-less rates. The objective is to build a lattice of risky rates on top of the risk-less rate process in an arbitrage-free manner by directly modelling credit spread components (transition and recovery risk). We generalise the Das \& Tufano ${ }^{[22]}$ (DT) model, where the spread term structure by rating class is modelled through three main components: risk neutral probability matrix, stochastic

\footnotetext{
${ }^{22}$ In fact, the structural approach assumes some explicit microeconomic model for the process that determines defaults or rating migrations of any single customers. A customer might be assumed to default if the underlying value of its assets falls below some specified threshold, such as the level of the customers' liabilities. The change in the value of a customer's assets in relation to various thresholds is often assumed to determine the change in its risk rating over the planning horizon. Structural approach models are Merton type models.

${ }^{23}$ Reduced-form models may differ depending on the procedure that is used, the input information required, the use of ratings-matrix and the recovery assumptions. As pointed out by Das and Sundaram (2000) ${ }^{[21]}$ There are three commonly used assumptions concerning recovery rates in the event of default: recovery of par, where the recovery amount is specified as a fraction of par value due at maturity; recovery of treasury, where recovery amount is specified as a fraction of value of a default-free bond with the same maturity; recovery of market value, where the recovery amount is specified as a fraction of the immediately-preceding market value.
} 
recovery rate and its correlation with interest rates. In the DT model the first component is aimed at estimating the transition risk; the second one, the recovery risk; the last one, the correlation between market and credit risk. In the integrated model different set-ups for different seniority classes are introduced within an arbitrage free framework. As the empirical evidence shows (see section 2 and 3 ) the mean recovery is mainly contingent on the seniority of debt rather than on the rating class alone (investment grade vs. non-investment grade in our analysis) as it is almost invariably assumed in all reduced model. The major contribution of this work it is to correct each STSRC through a spread contingent on the seniority of debt (SSD) within a unique arbitrage free framework. As a result, this model allows more variability in the spreads of risky debt. Moreover, by choosing different recovery rate processes for instruments within the same credit rating class, it allows variability of spreads to be instrument specific rather than rating class specific.

\subsection{The stochastic interest rate term structure model}

In the integrated model an interest term structure is assigned to each rating class $\mathrm{i}$ (where $0 \leq i \leq$ $K$, if we consider $K$ rating classes). The $i$-th interest rate $f_{i}$ at which cash flows are to be discounted is composed of forward risk-free interest rate plus a (forward) spread $s$ associated to the same rating class as shown below:

$\mathrm{f}_{\mathrm{i}}(t)=$ forward curve for rating class $-i=$ forward risk free $(t)+$ spread $-i$

In this context, the risk-free forward interest rate (stochastic) process can be modelled by using any interest rate term structure model like, for example, the Heath-Jarrow-Morton [1992] ${ }^{[32]}$ or the Black-Derman-Toy [1990] ${ }^{[9]}$ model. It is not the purpose of this paper to detail the risk neutral set up model formulation for the evolution of the interest rate free term structure, for which specialised literature may be addressed. More relevant to the present paper purposes is to illustrate how the spread is modelled for which the following paragraphs are devoted to.

\subsection{The stochastic spread term structure model}

Recovery rates, risk of default and the seniority type are relevant parameters for assessing credit risk. Therefore, in the integrated model, the spread is decomposed in its two main determinants a) recovery rates and $b$ ) default ${ }^{24}$ (transition) risk. Thus, in order to price the credit spread component of the interest rate term structure, both recovery rate and default variables need to be modelled. Let $q_{i k}$ be the (risk neutral) default rate ${ }^{25}$ (i.e., the rate at which default occurs). This rate may be either constant, or function of time-to-maturity of the security or of any other factor in the economy. The recovery rate will be denoted by $\phi$ and representing the fraction of the face value of the security that is recovered in case of default (by definition $0 \leq \phi \leq 1)$. Considering the influence of recovery and default rates on credit instruments, it is possible to consider a first simple relationship between these parameters and interest rate spreads. Let $r$ be the one period risk-less rate of interest, then the risk-neutral value $B$ of a credit risky bond maturing in a single period from now must be equal to the discounted value of expected cash flows in the future:

$$
B=\frac{q_{i k} \phi+\left(1-q_{i k}\right)}{1+r}
$$

where the parameters $q_{i k}$ and $\phi$ have been set to their risk-neutral values. On the other hand the price of the risky bond $B$ off the spread curve is given by:

\footnotetext{
${ }^{24}$ The default risk bearing also information on the type of seniority type

${ }^{25}$ The default rate being the rate at which default occurs
} 


$$
B=\frac{1}{1+r+s}
$$

By equating the right hand sides of Eq. (4.2.1) and Eq. (4.2.2) the required relationship between the spread $s$, which is the observed market spread for the generic security I, and the determinants of the spread may be derived. Solving by $s$ we obtain:

$$
s=\frac{q_{i k}(1-\phi)(1+r)}{1-q_{i k}(1-\phi)}
$$

In general, the actuarial estimation of the default rate is different from its risk neutral value, because the way through which the actuarial value is estimated is independent from the market price of that security. If, recalling eq. 2.1 , the actuarial default rate is, $\bar{q}_{i k}$ we have:

$$
s_{a c t}=\frac{\bar{q}_{i k}(1-\phi)(1+r)}{1-\bar{q}_{i k}(1-\phi)}
$$

where $s_{\text {act }}$ differs from s. To calibrate the statistical value of the default rate one can use spread market data. Given s, it is possible to render $q_{i k}$ risk neutral by summing to $\bar{q}_{i k}$ the adjustment factor $\pi$.

$$
s=\frac{\left(\bar{q}_{i k}+\pi\right)(1-\phi)(1+r)}{1-\left(\bar{q}_{i k}+\pi\right)(1-\phi)}
$$

Consequently $q_{i k}=\bar{q}_{i k}+\pi$. This approach allows coupling the model of the stochastic process for the interest rate term-structure to the market data, that is to say theoretical and empirical data.

From Eq. (4.2.3) it is possible to see that:

- the spread increases proportionally to the default rate $q_{i k}$ increase; this has a financial implication: as the default rate $q_{i k}$ increases the possibility of getting values far apart form the expected average value is higher. On the contrary, in the limiting case of default risk approaching zero $\left(q_{i k} \rightarrow 0\right.$; for $q_{i k}=0$ the recovery rate looses its meaning) the spread tends to zero $(s \rightarrow 0)$, allowing the certain value equal to the average

- the spread decreases proportionally to the recovery rate $\phi$ increase, which means that - in case of default - the higher is the chance of getting back the invested amount, the more limited fluctuations from the average price are got; in other words high recovery rates assure low credit risk. In the limiting case, approaching total recovery $(\phi \rightarrow 1)$ the spread still tends to zero $(s \rightarrow 0)$, in the ideal limiting case $s=0$ representing the evolution of a risk-less process

- when the default rate tends to one $\left(q_{i k} \rightarrow 1\right)$ and the recovery rate tends to zero $\quad(\phi \rightarrow 0)$ the spread tends asymptotically to become infinite.

Of course limiting cases are never reached but their study helps visualising the trend of the functional dependence of the spread from the default risk and recovery rate. In fact, as pointed out by $\operatorname{Das}^{[20]}$, Eq. (4.2.3) expresses the spread as a function of the composite variable $q_{i k}(1-\phi)$, for this reason the above formulation does not allow expressing the spread as a function of default risk and recovery rate independently. Therefore a more elaborated interest rate spread modelling is needed. Considering, for example, the HJM model, it is possible to observe that its structure allows for the required effective two-factor decomposition of credit spreads. Under 
the risk-neutral measure, the expected risky cash flows discounted at risk-less rates must be equal to the value of expected risk-less cash flows discounted at risky discount rates:

$$
\sum_{m=\frac{t}{\Delta}+1}^{\frac{T}{\Delta}}\left[E\left[\exp \left[-\Delta \sum_{j=\frac{t}{\Delta}}^{m-1} f_{i}(t, j \Delta)\right] * C_{d}(m)\right]=\exp \left[-\Delta \sum_{j=\frac{t}{\Delta}}^{\frac{T}{\Delta}-1}\left(f_{i}(t, j \Delta)+s_{i}(j \Delta)\right)\right] * 1\right.
$$

where $C_{d}(m)$ is the expected cash flow of the risky bond in case of default at the time step- $m$ before maturity and $l$ is the cash flow in case of non-default. In order to render $C_{d}(m)$ in explicit form it is necessary to define the cumulative and one-period default probabilities associated to the rating class of the instrument at any given time, and to consider the recovery rate at the corresponding default time. Including the default risk, the recovery rate and the credit seniority information in $C_{d}(m)$, by means of $E q$. (4.2.3) it is possible to estimate the determinants of the interest rate term structure spread associated to the rating class $I$ contingent to the seniority of debt. In order to develop a consistent framework - since for the interest rate term structure model a risk-neutral world is assumed, the actual transition probabilities (estimated by using the mortality approach ${ }^{26}$ described in section 2 ) have to be risk-neutral adjusted. After having obtained the risk-neutral set up for the evolution of the term structure of interest rates, the integrated model derives the risk-neutral probabilities of the transition process to default. Summarising, we will first correlate the interest rate term with one recovery rate structure, then, we will generalise the results by considering s seniority type thus including the spread correction due to the recovery dependence on the seniority. Following this set up a new stochastic framework for the arbitrage-free pricing of risky debt is depicted. This framework is illustrated through the following three steps:

1. first construct a one period risk neutral probability matrix for each seniority type

2. then extend to a multi-period framework through the definition of a cumulative risk-neutral transition matrix which allows the obligor to default at any point in time

3. third estimate the STSRC contingent to the seniority of debt

\subsubsection{Risk-neutral probability transition matrix}

One of the key points in which the integrated model departs from other models is in the spread dependence assumption of both the recovery rate on seniority s and of the rating class. In general, in the integrated model, the recovery rate is assumed to follow any "reasonable" distribution. We suggest calibrating the model by using a beta distribution in according to the empirical evidence described in the second section. In practice, any value for the recovery rate is possible with a non zero probability. The probability density function of the beta distribution is given by:

$$
g_{s m}\left(\phi_{s m}, \alpha_{s m}, \beta_{s m}\right)=\left\{\begin{array}{ll}
\frac{\Gamma\left(\alpha_{s m}+\beta_{s m}\right)}{\Gamma\left(\alpha_{s m}\right) \Gamma\left(\beta_{s m}\right)} \phi_{s m}^{\alpha_{s m}-1}\left(1-\phi_{s m}\right)^{\beta_{s m}-1} & \text { for } 0<\phi_{s m}<1 \\
0 & \text { for } \phi_{s m}<0 \text { and } \phi_{s m}>1
\end{array} \text { with } \mathrm{s}=1, \ldots, 5\right.
$$

where $\phi_{s m}$ represents the fraction of recovery at time $\mathrm{m}$ associated to the seniority type $\mathrm{s}$, $g_{s m}($.$) represents the probability associated to that recovery rate (belonging to the s seniority$ class); the seniority type range is between 1 and 5 because the considered seniority classes are

\footnotetext{
${ }^{26}$ The statistical migration matrix is an input in this pricing model. One can also use other approach, like the S\&P or Moody's method of estimating the migration matrix
} 
5: senior secured, senior unsecured, senior subordinated, subordinated, junior subordinated. Moreover $\Gamma$ stands for the gamma distribution and $\alpha_{s m}$ and $\beta_{s m}$ are two generic parameters which depend from both the seniority and the time. Equation 4.2.2.1 has the required property that 0 and 1 bound the recovery ${ }^{27}$. If $\mu_{s m}=31,73 \%$ and $\sigma_{s m}=22,06 \%$, as for the subordinated non-investment grade bond (see table 1), the pdf of the beta distribution is depicted in figure 1.

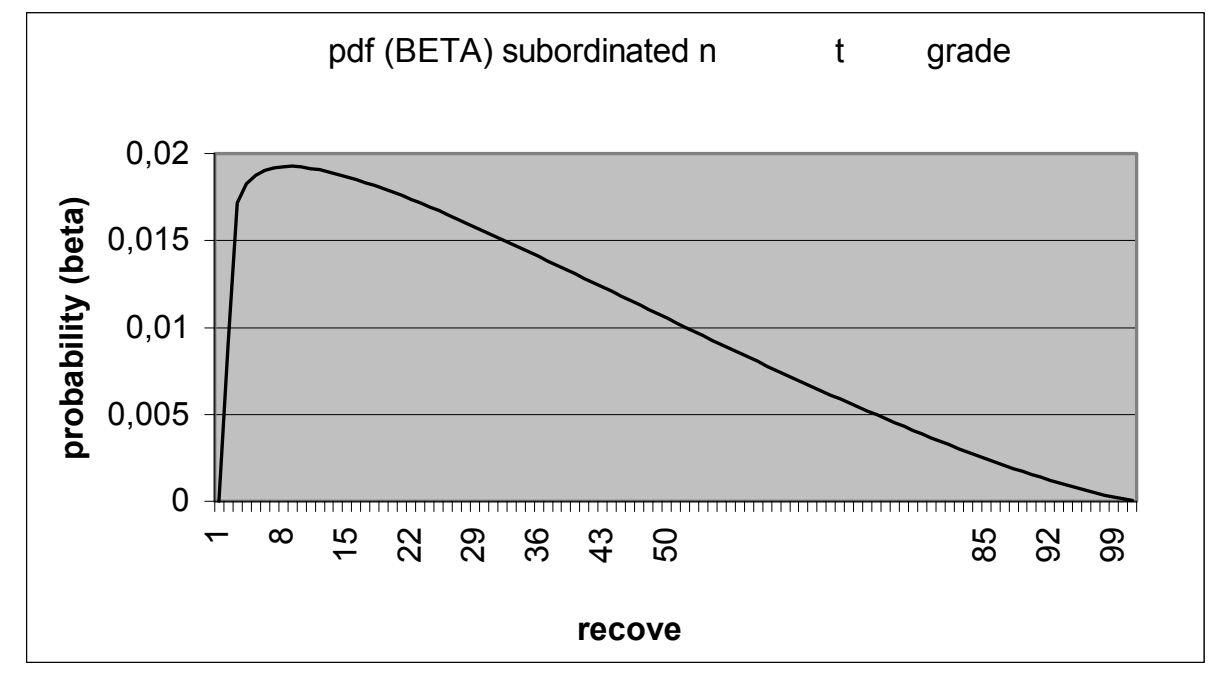

Figure 1 Beta Distribution

The figure illustrates the high degree of randomness present in recovery rates.

The model objective is to develop a risk neutral lattice for pricing risky debt. In order to render the forward interest and recovery rate process tractable numerically, the corresponding state space $^{28}$ could be "discretised" consistently through both the (discretised) time structure, $\mathrm{m}$, and through the "shock" vectors $\mathrm{v}^{29}$, for the risk free term structure process, and $\mathrm{z}$, for the recovery rate process. To implement the model, we make the standard discrete time assumption that $\mathrm{v}$ and $\mathrm{z}$ are binomial random variables. In particular, we assume that both $\mathrm{v}$ and $\mathrm{z}$ takes on the value \pm 1 with probability 0,5 :

$$
v=\left[\begin{array}{l}
+1 \\
+1 \\
-1 \\
-1
\end{array}\right] ; \quad z=\left[\begin{array}{l}
+1 \\
-1 \\
+1 \\
-1
\end{array}\right]
$$

Consequently, "discretising", the recovery rate vector, function of each seniority type s at the given time $m$, becomes:

\footnotetext{
${ }^{27}$ The parameters can be computed since the mean $\mu_{\mathrm{sm}}$ and variance $\sigma_{\mathrm{sm}}^{2}$ of a beta distribution are given by: $\mu_{s m}=\frac{\alpha_{s m}}{\alpha_{s m}+\beta_{s m}}$ and $\sigma_{s m}^{2}=\frac{\alpha_{s m} \beta_{s m}}{\left(\alpha_{s m}+\beta_{s m}\right)^{2}\left(\alpha_{s m}+\beta_{s m}+1\right)}$ where $\mu_{s m}$ and $\sigma_{s m}$ are the mean and standard deviation of the actual empirical distribution of credit recovery belonging to seniority debt type-s.

${ }^{28}$ The state-space is defined as the ensemble of all possible states related to the stochastic process.

${ }^{29}$ If, for example, the HJM model is used to build the risk neutral set up for the estimation of the risk free term structure, $\mathrm{v}$ represent the random variable of the underlying stochastic process, i.e.: $f(t+m, T)=f(t, T)+a(t, T) m+\sigma(t, T) v \sqrt{m}$, where $\alpha$ and $\sigma$ represent respectively both the drift and the volatility of the process. In a discrete time set up, periods are taken to be of length $m>0$, thus a typical time point, $t$, has the form $1 \mathrm{~m}$ for integer 1 .
} 


$$
\phi_{s m}(z)=\left[\begin{array}{c}
+\phi_{s m} \\
-\phi_{s m} \\
+\phi_{s m} \\
-\phi_{s m}
\end{array}\right] ; \mathrm{s}=1, \ldots, 5
$$

From now on, to reduce the notational burden, we suppress the dependence from $\mathrm{z}$ and in the remainder we will consider $\phi_{s m}(z)=\phi_{s}(m)$. We will remark the time dependence because we will allow in our model to choose different beta distribution parameterisation in different time, like for example, in different economic cycle. In a discrete time set up, in order to consider a consistent and integrated risk-neutral framework, it is necessary to correlate the state space recovery rates structure with the forward rates term structure at any given time. Let us define $\rho$ as the (empirical) correlation between the term interest rate structure and the recovery rate ${ }^{30}$, the

$$
\text { assumed joint distribution is: } \mathbf{f}^{\prime}(\mathbf{v}, \mathbf{z})= \begin{cases}(+1,+1), & \text { with prob. }\left(\frac{1+\rho}{4}\right) \\ (+1,-1), & \text { with prob. }\left(\frac{1-\rho}{4}\right) \\ (-1,+1), & \text { with prob. }\left(\frac{1-\rho}{4}\right) \\ (-1,-1), & \text { with prob. }\left(\frac{1+\rho}{4}\right)\end{cases}
$$

Moreover, let us define $\rho^{\prime}$ as the risk neutral probability vector ${ }^{31}$ collecting the states probabilities of each branch of the lattice: $\rho^{\prime}=\left[\begin{array}{c}\frac{1+\rho}{4} \\ \frac{1-\rho}{4} \\ \frac{1-\rho}{4} \\ \frac{1+\rho}{4}\end{array}\right]$.

For computational needs and for notation ease, it is useful to introduce another concept before getting the final explicit functional form for the forward spreads: the state-prices ${ }^{32}$. The state price (denoted by the variable $\mathrm{w}(\mathrm{m})$ ) at time $m+1$ evaluated at time- $m$, is defined as the price at time- $m$ times the risk-neutral probability $\rho$ ' of being in that state at the time- $m$ discounted at the risk-free interest rate, i.e.:

$$
w(m \Delta)=\rho^{\prime} \cdot w[(m-1) \Delta] \cdot \frac{1}{1+f_{m-1, m}}
$$

where the state prices are considered as four-dimensional vectors (corresponding to the four possible states defined by the double stochastic structure) for each seniority and $f_{m-1, m}$ is the forward rate between time $\mathrm{t}=(m-1) \Delta$ and time $t=m \Delta$. Both the interest rate term structure and

\footnotetext{
${ }^{30}$ The definition of the parameter $\rho$ allows having one more degree of freedom, which enables to perform the proper recovery rates and interest rates correlation choice according to the overall economy time-scale considered in the model.

${ }^{31}$ The vector is risk neutral by construction having assumed that $\mathrm{v}$ and $\mathrm{z}$ takes on the value \pm 1 with probability 0,5 .

${ }^{32}$ As pointed out by Das and Sundaram (2000) [29] "State prices are the current value of a security that pays off a dollar in a single specific state in the future and zero in all other states. For example, if there are only two possible states ("up" and "down") at the same time in the future, then the state price of the "up" state would be the value of a dollar received in that state times the risk neutral probability of that state, discounted to the present, using a risk-less discount rate. State prices are useful since they allow to compute the price of any security by multiplying the payoffs of the security by state prices in each node (state), and then add these values up. Of course at time 0 the state price is simply unity. i.e. $\mathrm{w}(0)=1$ "
} 
the recovery rate structure are implied in the definition of the state price, track of them can be found in the discount and probability factors, respectively. The cash-flow at time step- $m$ is a function of recovery rates as well as transition rates. While recovery rates are correlated to the risk-neutral interest rate structure, the transition probabilities have to be rendered risk-neutral in order to preserve the overall framework consistency. For this purpose, as generally described in paragraph 4.2, it is necessary to introduce rating class $i$ and seniority s specific adjusting factors to the empirical transition probabilities defined for any time step- $m \pi_{i}^{s}(m)$. Let us consider the one-period transition from a generic time- $m$ to time $(m+1)$; this is performed by defining the unknown quantities $\pi_{i}^{s}(m)$ referred to the $i$-th rating class and to the $s$-th seniority type ${ }^{33}$ of the credit instrument at time step- $m$.

$$
\begin{aligned}
Q^{s}(m) & =\left\{q_{i j}^{s}(m)\right\}=\left[\begin{array}{cccc}
q_{11}^{s}(m) & . . & . . & q_{1 K}^{s}(m) \\
q_{21}^{s}(m) & q_{22}^{s}(m) & . . & q_{2 K}^{s}(m) \\
. . & . . & . . & . . \\
0 & 0 & . . & 1
\end{array}\right] \equiv \\
& \equiv\left[\begin{array}{cccc}
1-\left(1-\pi_{1}^{s}(m)\right) \cdot \bar{q}_{11}(m) & \bar{q}_{12}(m) \cdot \pi_{1}^{s}(m) & . . & \bar{q}_{1 K}(m) \cdot \pi_{1}^{s}(m) \\
\bar{q}_{21}(m) \cdot \pi_{2}^{s}(m) & 1-\left(1-\pi_{2}^{s}\right) \cdot \bar{q}_{22}(m) & . . & \bar{q}_{2 K}(m) \cdot \pi_{2}^{s}(m) \\
. . & . . & . . & . . \\
0 & 0 & . . & 1
\end{array}\right]
\end{aligned}
$$

where $Q^{s}(m)$ is the risk neutral representation of $\bar{Q}(m)$, when incorporating the seniority type effect in the transition matrix by rating class, as shown in the generic element $q_{i j}^{s}(m)$, which, by construction, explicitly consider the adjustment factor $\pi_{i}^{s}(m)$. Invoking the definition of state price, for the credit instrument of seniority type- $s$ being in class- $i$ at time- $m$, the following condition, in a risk neutral world, must be satisfied:

$w(m \Delta) \cdot E\left[C^{s}(m)\right]=\frac{1}{1+f_{m M}^{a c t}+s}(4.2 .1 .7)$; where $f_{m M}^{a c t}$ is the actual forward interest in the period between time- $m$ and maturity (time- $M$ ), $\mathrm{s}$ is the market spread and the expected cash flow at time- $m$ for the bond of rating class $-i$ and seniority type-s is determined by: $E\left[C_{i}^{s}(m)\right]=\left[q_{i 1}^{s}(m), q_{i 2}^{s}(m), \ldots, q_{i K}^{s}(m)\right] \cdot\left[1, \quad 1, \quad \ldots, \quad \phi_{s}\right]$

Eq. (4.2.1.7) and Eq. (4.2.1.8) provide the solutions for the unknown $\pi_{i}^{s}(m)$ associated to the rating class- $i$ and seniority type-s by calibrating those equations with the (average) market spread of the considered risky debt. In fact, making use of simple algebra, it is possible to show that $E q$. (4.2.1.7) is the generalisation of $E q$. (4.2.5) when considering the assumptions of the suggested integrated pricing model. Applying the above-mentioned market price calibration it is possible to find all the adjustment factors to get the risk neutral transition matrix for seniority type-s at time $t$. Five transition matrices for each rating class correspondent to the five seniority types are generated. Therefore the model can be split into five parallel models yielding specific information on the seniority for any rating class, at any time step. This information is then embedded in the final expression of the spread related to the seniority type. At this stage it is important to observe that, according to the data in table 1, default rates are not affected by the credit instrument dependence on seniority, while the recovery rate does. Within this unified risk

\footnotetext{
${ }^{33}$ One reason behind the choice of $\mathrm{K}$ rating class and s seniority type is that there are well documented tables of default frequencies for standard ratings but there is not enough data in all cases to distinguish between different seniority types. Another reason is that while ratings are subject to random changes the seniority class remains unchanged during the life of an asset
} 
neutral framework it is possible to measure the contribution of the seniority of a credit issue to the risk neutral spread curve.

\subsubsection{Multiple time horizon}

Up to now the attention was focussed on those variables, assumptions and parameters that have a direct impact on credit risk, without explicitly considering the time at which those quantities have been evaluated or defined. Another basic managerial aspect of credit risk is the time horizon of the risk measure. This measure of risk of a financial instrument is a critical issue. In fact, in this case the problem of extrapolation, or interpolation, has to be faced in order to achieve the correct estimation of migration probabilities in the multiple time-horizon. Let us consider the one-period probabilities $q_{i j}^{s 0}(\mathrm{~m})$ as the probability of migrating from rating class- $i$ to rating class- $j$ in the time interval between time step- $(m-1)$ and step-m with respect to a generic recovery rate structure s. Actually, the probabilities previously considered in the transition matrices elements at the generic time step- $m$ are regarded as cumulative probabilities; the actual cumulative probabilities are obtained by the one step probabilities by a recursive procedure. Under the assumption that the one period migration probabilities at subsequent time steps are independent, it is possible to obtain the actual rating class transition probabilities, from any class $-i$ to any class- $j$, at the subsequent time step by multiplying the actual migration matrix at time- $m$ with the one at time $m+1$. This procedure may be applied recursively yielding for the actual transition matrix at time $T$. Applying the risk-neutral adjustment procedure at any time step as outlined in previous paragraph, the risk-neutral transition matrix at time period $m+1$ is directly derived. It is important to point out that this structure allows embedding in any transition probability at the given time- $m$ all the information on transition probabilities at previous time steps (maintaining probabilities independence), therefore the single one-period transition probability $q_{i j}^{s}(m)$ keeps the information on $q_{k l}^{s}(n)$ for all states $k, l$ and for all times steps- $n(n<m)$. In particular, the default probabilities $q_{i k}^{s}(m)$ contain the information on the previous time step transition probabilities. This feature distinguishes the integrated model form the other reduced models outlined in section 1,2 and 4 . The transition probability can change significantly over time. An investment grade has a higher chance of downgrade than of upgrade and vice versa (mean reversion in credit ratings). This means that in the high rated firms transition risk (and default probability in particular) increase over time and, by contrast, high yield risky debt that do not default, are more likely to improve than deteriorate in credit quality, thus showing a decreasing default probability over time.

\subsubsection{One-period and cumulative transition probabilities}

Before deriving the formula for the spread curve it is interesting to focus on transition probabilities. Provided that $K$ rating classes are considered, $q_{i k}^{s 0}(m \Delta)$ is defined as the oneperiod default probability over the period from $[(m-1) \Delta, m \Delta]$ associated to the state $I$ and generic seniority $s$, i.e. the probability of migration from the rating class $i$ to class $K$ (corresponding to the default state). The cumulative probability of default at the time period- $m$ $(t=m \Delta)$ is defined as $q_{i k}^{s}(m \Delta)$, and it is a function of the previous-time cumulative probability ${ }^{34}$ and the one-period default probability ${ }^{35}$ as follows:

$$
q_{i K}^{s}(m \Delta)=q_{i K}^{s}((m-1) \Delta)+\left[1-q_{i K}^{s}((m-1) \Delta)\right] \cdot q_{i K}^{s 0}(m \Delta)
$$

\footnotetext{
${ }^{34}$ The previous time cumulative probability is the probability of having got default until the previous time step.

${ }^{35}$ The one-period default probability is the probability of getting to default between $(\mathrm{m}-1) \Delta$ and $\mathrm{m} \Delta$
} 
Conversely, the one period probability of default in the period indexed by $m$ may be expressed

as: $q_{i K}^{s 0}(m \Delta)=\frac{q_{i K}^{s}(m \Delta)-q_{i K}^{s}((m-1) \Delta)}{1-q_{i K}^{s}((m-1) \Delta)}$

These definitions are useful to compute expected cash flows over time for a zero coupon risky bond. Since $q_{i K}^{s}(m \Delta)>0$, and the cumulative probability of default must be increasing:

$q_{i K}^{s}(m \Delta)-q_{i K}^{s}((m-1), \Delta)>0$

then, default probabilities lie in the range $[0,1]$ as required. In this formalisation it is important to point out that by means of the procedure outlined above, the risk-neutral adjusted transition probabilities to default transmit the information of all the actual transition probabilities. At this stage all the information required for deriving the spread structure as a function of its determinants has been derived and may be embedded into the cash flow evaluation. With reference to Eq. (4.2.6) and Eq. (4.2.1.8), the expected cash flow at the $m$-th time period for the given seniority class- $s$ in its explicit form is:

$$
E\left[C_{d}^{s}(m)\right]=1 \cdot\left[1-q_{i k}^{s}((m-1) \Delta)\right] \cdot q_{i k}^{s 0}(m \Delta) \cdot \phi_{s}(m \Delta, n)
$$

which also generalise Eq. (4.2.1). As pointed out in the multiple time horizon approach, in the integrated model the one-period probabilities are given by the first transition probability, and the cumulative probabilities are derived recursively. The philosophy of the integrated model appears evident also at this stage since the strict correlation between the underlying model structure and the empirical data is assured at each step of the formulation: theory and actual data are interwoven in order to assure adherence between the theoretical process and the market dynamics. Recalling Eq. (4.2.3.4) it is possible to rewrite Eq. (4.2.6) in the following way:

$$
\sum_{m=\frac{t}{\Delta}+1}^{\frac{T}{\Delta}-1}\left[E\left(\exp \left[-\Delta \sum_{j=\frac{t}{\Delta}}^{m-1} f_{i}(t, j \Delta)\right] C^{s}(m)\right]=\exp \left[-\Delta \sum_{j=\frac{t}{\Delta}}^{\frac{T}{\Delta}-1}\left(f(t, j \Delta)+s p_{i}^{s}(j \Delta)\right)\right] ; \forall i\right.
$$

Making use of both the definition of state prices and cash-flow in case of default (see Eq. 4.2.1.7) at any time-step- $m$ Eq. (4.2.3.5) becomes:

$$
\left.\sum_{m=\frac{t}{\Delta}+1}^{\frac{T}{\Delta}-1}\left[\sum_{n=1}^{m} w(m \Delta, n)\left[1-q_{i k}^{s}(m-1) \Delta\right)\right] q_{i k}^{s 0}(m \Delta) \phi_{s}(m \Delta, n)\right]=\exp \left[-\Delta \sum_{j=\frac{t}{\Delta}}^{\frac{T}{\Delta}-1}\left(f(t, j \Delta)+s p_{i}^{s}(j \Delta)\right)\right] ; \forall i
$$

\subsection{Spread term structure by rating class contingent to the seniority of debt}

The term structure of forward credit spreads estimation is the problem to be solved in last step of the process. For any rating class and seniority type the following spread, $s p$, set is given $\left\{s p_{i}^{s}(t)\right\} i=1, \ldots, K ; \mathrm{s}=1, \ldots, 5 ; t<T$

In order to give the spread curve in its explicit form it is necessary to consider its integral formulation. The spread curve evaluated at time $t$ for a given rating class- $i$ is defined by all spreads computed at consequent time steps within the bond life-span, specifically in the time interval $[\mathrm{t}, \mathrm{T}]$. Let us consider Eq. (4.2.3.6) and define the integral spread curve $\mathrm{S}(\mu, \mathrm{M})$ between the $\mu$-th and the $M$-th period (corresponding to any given time $\tau \in[\mathrm{t}, \mathrm{T}]$ and maturity $\mathrm{t}=\mathrm{T}$, respectively) 


$$
\begin{aligned}
& S P_{i}^{s}\left(\frac{\tau}{\Delta}, \frac{T}{\Delta}\right) \equiv S P_{i}^{s}(\mu, M) \equiv \sum_{j=\frac{t}{\Delta}}^{\frac{T}{\Delta}-1} s p_{i}^{s}(j \Delta) \equiv \\
& \left.\equiv-\frac{1}{\Delta} \cdot \operatorname{Ln}\left\{\sum_{m=\tau / \Delta+1}^{T / \Delta}\left[\sum_{n=1}^{m} w(m \Delta, n)\left[1-q_{i K}^{s}(m-1) \Delta\right)\right] q_{\mathrm{iK}}^{\mathrm{s} 0}(m \Delta) \phi_{s}(m \Delta, n)\right]\right\}-\sum_{j=\frac{\tau}{\Delta}}^{\frac{T}{\Delta}-1} f(\tau, j \Delta)
\end{aligned}
$$

In order to derive the spread curve at time step- $\mu$ the following differential relation is used $s p_{i}^{s}\left(\frac{\tau}{\Delta}\right) \equiv s p_{i}^{s}(\mu) \equiv S P_{i}^{s}(\mu, M)-S P_{i}^{s}(\mu-1, M)$

Finally, referring to Eq. (4.3.2) the forward interest rate spread is determined as:

$$
s p_{i}^{s}(\mu) \equiv-\frac{1}{\Delta} \operatorname{Ln}\left\{\frac{\left.\sum_{m=\tau / \Delta+1}^{T / \Delta}\left[\sum_{n=1}^{m} w(m \Delta, n)\left[1-q_{i K}^{s}(m-1) \Delta\right)\right] q_{i K}^{s 0}(m \Delta) \phi_{s}(m \Delta, n)\right]}{\left.\sum_{m=\tau / \Delta}^{T / \Delta}\left[\sum_{n=1}^{m} w(m \Delta, n)\left[1-q_{i K}^{s}(m-1) \Delta\right)\right] q_{i K}^{s 0}(m \Delta) \phi_{s}(m \Delta, n)\right]}\right\}-\left[\sum_{j=\tau}^{\frac{T}{\Delta}-1} f(\tau, j \Delta)-\sum_{j=\frac{\tau}{\Delta}-1}^{\frac{T}{\Delta}-1} f(\tau-\Delta, j \Delta)\right]
$$

The last-period forward spread between time $T$ and time $T+\Delta$ relative to the $i$-th rating class and adjusted for the seniority $\mathrm{s}$ is denoted by $s p_{i}^{s}(T)=s p_{i}^{s}(M)$, by computing node- $T$ on the tree of the interest forward rate structure, the spread is derived considering the last period expected cash-flow in case of default without considering previous cash-flow events. Referring to Eq. (4.3.4) it is straightforward to derive the last-period spread as follows $\exp \left[-\Delta s p_{i}^{s}(T)\right]=E\left[1-\frac{q_{i K}^{s}(T+\Delta)-q_{i K}^{s}(T)}{1-q_{i K}^{s}(T)}\left(1-\phi_{s}(T+\Delta)\right)\right], \forall i$

\section{Applications}

Our model requires easily available information as input, namely: the risk-free yield curve, the term structure of credit spreads for each rating class, the statistical transition matrix and both mean and standard deviation of the recovery rate by seniority of debt. The most important and useful resultant model information is: risk neutral transition matrix and risk neutral spread term structure are both contingent on the seniority of debt. Moreover, the bivariate lattice, thorough which the STSRC and SSD has been estimated, was built by correlating riskless interest rates and recovery rates thus considering the integration between market and credit risk. Using this information, the following products, among others, are priced by generating the necessary cash flows at each node on the lattice and discounting the cash flows back by multiplication of the state prices to obtain present values on plain-vanilla risky debt of any rating class and any seniority of debt. Our model performs quite well to price rating-sensitive debt since the rating transition matrix provides risk-neutral information on rating changes (adjusted to the seniority) which can be directly used to generate cash flows at each node on the tree. For spread-adjusted notes, the coupon may also be indexed to the spread at each node, this is achieved by computing the forward spread at each node on the lattice and, since the price of the risky debt is known at each node, and so is the riskless rate, it is quite simple to compute the credit spread at each node as well. It is also possible to price spread option since cash flows may be generated at each node by comparing the spread at the node with the strike rate. For total return swaps, since the price of any underlying risky bond is computable at each node on the tree, the total return on the bond may also be easily calculated. Although the model is rich and flexible enough to price many credit assets, both plain vanilla and derivative, we think is particularly appropriate to price defaultable loans and corporate bonds. 


\section{Conclusions}

The stochastic spread structure model considered within the integrated model allows taking into account effects due to rating transitions (including default events) and recovery rates depending on seniority. The overall procedure allows discriminating the effects of the credit instrument belonging to a specified rating class at any given time; actually fixing the time step in the forward interest rate term structure, $k-1$ spreads corresponding to the defined rating classes are derived. More specifically, this model is aimed at computing the spread for credit instruments belonging to a defined rating class and having a specified seniority, so that to discriminate the information relative on the given seniority. This framework allows depicting the effects on spread curves due to the rating class, and -for any given rating class, the effects due to the different seniority types using the risk neutral arbitrage set-up.

Further research on this area will be devoted both on considering the influence of the economic cycle and the supply/demand for defaulted assets on the estimation of recovery contingent to seniority and analyse the structural (firm related) interdependencies between recovery rates and default probability. Moreover the issue of default correlation and its impact on pricing risky debt should also be investigated. Finally, from a practical point of view, there are at least two other relevant issues that need to be carefully taken in consideration in future work, namely liquidity risk and parameter calibration. Our intuition is that we need an integrated pricing and risk model to exploit in a coherent framework the risk and capital management banking problem.

\section{References}

[1] Acharya V.V., Das S. R., Sundaram R. K., Pricing credit derivatives with rating transitions, Financial Analysts Journal, (3)(2002)28-42.

[2] Altman E.I., Caouette J.B., Narayanan P., Managing credit risk: the next financial challenge, Ed. John Wiley \& Sons, Inc., 1998.

[3] Altman E.I., Pompeii J., Market size and investment performanceof defaulted bonds and bank loans, Salomon Center NYU, 2002.

[4] Altman E.I., Kishore V.M., Almost everything you wanted to know about recoveries on defaulted bonds, Financial Analysts Journal, (6)(1996)57-63.

[5] Altman E.I., Onorato M., Pastorello A., A general framework for credit risk models, Working Paper, Trondaim 2000.

[6] Basle Committee on Banking Supervision, Credit risk modelling: current practice and applications, 1999.

[7] Basle Committee on Banking Supervision, Principles for credit risk management, 1999.

[8] Basle Committee on Banking Supervision, A new capital adequacy framework, 1999.

[9] Black F., Derman E., and Toy W., A one-factor model of interest rates and its application to treasury bond options, Financial Analysts Journal, (1)(1990)33-39.

[10] Carty L.V., Lieberman D., Corporate bond defaults and default rates 1938-1995, Moody's Investor Service, Global Credit Research, Special Report, 1996.

[11] Carty L.V., Lieberman D., Defaulted bank loan recoveries, Moody's Investor Service, Global Credit Research, Special Report, 1996.

[12] Carverhill A., A simplified exposition of the Heath, Jarrow, and Morton model, Stochastics, 1995, pp. 227-240.

[13] Castle K., Keisman D. and Yang R. "Suddenly structure mattered: insights into recoveries from defaulted debt" Standard \& Poor's, Credit Weekly, 2000.

[14] Cooper I. A. and Mello A. S., Default risk and derivative products," Applied Mathematical Finance (3)(1996)53-74.

[15] CreditMetrics ${ }^{\mathrm{TM}}$, Technical document JP Morgan, 1997. 
[16] Credit Suisse Financial Products, CreditRisk $+{ }^{\mathrm{TM}}$, A credit risk management framework, 1997.

[17] Crosbie P., Modelling default risk, KMV Corporation, 1998.

[18] Crouhy M., Galai D., Mark R., A comparative analysis of current credit risk models, Journal of Banking \& Finance (24)(2000)59-117.

[19] Dai Q. and Singleton K., Specification analysis of affine term structures models, Research Paper, Graduate School of Business, Stanford University, 1998.

[20] Das S.R., Credit risk derivatives, Journal of derivatives, (3)(1995)7-23.

[21] Das S.R., Sundaram R.K., A discrete time-approach to arbitrage-free pricing of credit derivatives, Working Paper, 1999.

[22] Das S.R., Tufano P., Pricing credit sensitive debt when interest rates, credit ratings and credit spreads are stochastic, Journal of Financial Engineering, (5)(1996)161-198.

[23] Duffee G., Estimating the price of default risk, Review of Financial Studies, (1)(1999)197-226.

[24] Duffie G., "The relation between treasury yields and corporate bond yield spreads", Journal of Finance, 1998.

[25] Duffie D., Credit swap valuation, Financial Analysts Journal, (1)(1999)73-87.

[26] Duffie D. and Lando D., The term structure of credit spreads with incomplete accounting information, Graduate School of Business, Stanford University, 1997.

[27] Duffie D., Pan J., and Singleton K., Transform analysis and option pricing for affine jump diffusions, Graduate School of Business, Stanford University, 1998.

[28] Duffie D., and Singleton K., Modelling term structure of defaultable bonds, Review of Financial Studies, 1996.

[29] Gordy M.B., A comparative anatomy of credit risk models, Journal of Banking and Finance (24)(2000)119-149

[30] Goupton G.G., Stein R.M., Loss Calc ${ }^{\mathrm{TM}}$ Moody's model for predicting loss given default, Moody's Investor Services, 2002.

[31] Harrison M. and Kreps D., Martingales and arbitrage in multiperiod security markets, Journal of Economic Theory (20)(1979)381-408.

[32] Heath D., Jarrow R., and Morton A., Bond Pricing and the Term Structure of Interest Rates: A Methodology for Contingent Claims Valuation, Econometrica (60)(1992)77-106.

[33] Huge B. and Lando D., Swap pricing with two-sided default risk in a rating-based model, Working Paper, University of Copenhagen 1999.

[34] Hull J. and White A., The impact of default risk on the prices of options and other derivative securities, Journal of Banking and Finance (1995)(19)299-322.

[35] Jarrow R.A., Lando D., Turnbull S.M., A Markov model for the term structure of credit risk spreads, The Review of Financial Studies (2)(1997)481-523

[36] Jarrow R., Turnbull S.M., The intersection of market and credit risk, Journal of Banking and Finance, 2000.

[37] Jarrow R. and Turnbull S., Pricing options on financial securities subject to default risk, Journal of Finance (50)(1995)53-86.

[38] Kealhofer S., Portfolio Management of Default Risk, KMV Corporation, 1998.

[39] KMV Corporation, Credit Monitor Overview, San Francisco, California, 1993.

[40] Koyluoglu H.U., Hickman A., Reconcilable differences, Risk (10)(1998)56-62.

[41] Litterman R. and Iben T., Corporate bond valuation and the term structure of credit spreads, Journal of Portfolio Management, (2)(1991)52-64.

[42] Longstaff F.A., Schwartz E.S., Valuing credit derivatives, Journal of Fixed Income, (5)(1995)6-12

[43] Longstaff F.A., Schwartz E.S., A simple approach to valuing risky fixed and floating rate debt, Journal of Finance (50)(1995)789-819.

[44] Merton R., On the pricing of corporate debt: the risk structure of interest rates, Journal of Finance (29)(1974)449-470.

[45] Neilsen 1., Saa-Requejo J., and Santa-Clara P., Default risk and interest rate risk: the term structure of default spreads," Working Paper, INSEAD, Fontainebleau, France, 1993.

[46] Ong M.K. "Internal Credit Risk Models", Risk Books, 1999. 
[47] Rosen D. Enterprise credit risk management, Algorithmics publication, 2002

[48] Saunders A., Credit risk measurement, value-at-risk and other paradigms, Ed. John Wiley \& Sons, Inc., 2001.

[49] Schonbucher, P.J., Term-structure modelling of defaultable bonds," Review of Derivatives Research (2)(1998)161-92.

[50] Shimko, D., N. Tejima, and D. Van-Deventer, The pricing of risky debt when interest rates are stochastic, The Journal of Fixed-Income (3)(1993)58-65.

[51] Skora, R. Credit modelling and credit derivatives: Rational Modelling, working paper, Skora and Co, Inc, 1998.

[52] Vasicek O., Probability of loss on loan Portfolio, KMV Corporation, 1987)

[53] Wilson T., Portfolio Credit Risk (I), Risk 10, N 9, 1997.

[54] Wilson T., Portfolio Credit Risk (II), Risk 10, N 10, 1997. 